\title{
Diferenças Genéticas e Estimação de Coeficientes de Herdabilidade para Características Morfológicas em Fêmeas Zebus e $F_{1}$ Holandês x Zebu ${ }^{1}$
}

\author{
Gerson Barreto Mourão², José Aurélio Garcia Bergmann ${ }^{3}$, Fernando Enrique Madalena ${ }^{3}$, Marcos \\ Brandão Dias Ferreira4
}

RESUMO - O objetivo deste trabalho foi averiguar possíveis variações nas mensurações morfológicas entre reprodutrizes zebus dos grupos genéticos (GG) Indubrasil, Nelore e Tabapuã e entre os GG de suas filhas $\mathrm{F}_{1}$ Holandês x Indubrasil (HxI), Holandês x Nelore $(\mathrm{HxN})$ e Holandês x Tabapuã (HxT), além de estimar coeficientes de herdabilidade $\left(\mathrm{h}^{2}\right)$ para estas características. O método dos quadrados mínimos foi usado para verificação das diferenças nas características morfológicas entre os GG e para obtenção de estimativas de $\mathrm{h}^{2}$ pela regressão filha-mãe (RFM). O método REML foi também usado para estimar $\mathrm{h}^{2}$. Os dados foram coletados em 183 vacas zebus e 273 novilhas $\mathrm{F}_{1}$ Holandês $\mathrm{x}$ Zebu. $\mathrm{O}$ GG das fêmeas $\mathrm{F}_{1}$ foi importante para sua inscrição no livro de controle e registro genealógico da raça Girolando, pois os GG obtiveram diferentes pontuações nas características de classificação total para tipo, aparência geral e caracterização leiteira. Para estas três características, as mestiças HxI obtiveram melhor pontuação que as HxN e as novilhas HxT, pontuação intermediária. Entre as características morfológicas, as larguras entre as tuberosidades ilíacas e as isquiáticas, o comprimento da garupa, a profundidade torácica, a altura na cernelha e o peso corporal não apresentaram diferenças entre os três GG zebus e entre suas progênies $\mathrm{F}_{1}$ Holandês x Zebu. As estimativas de herdabilidade variaram de 0,09 (profundidade torácica) a 0,59 (comprimento da orelha) pela RFM e de 0,09 a 0,89 (mesmas características) pelo método REML. Os resultados indicam que, em sistema de cruzamento com a raça Holandesa, para a produção de novilhas $\mathrm{F}_{1}$ para venda, o GG Indubrasil produziu animais melhor caracterizados, como da raça Girolando, os quais, possivelmente, teriam maior apelo comercial que os dos GG Tabapuã e Nelore. Adicionalmente, as estimativas de herdabilidade indicaram que a seleção para características morfológicas levaria a ganhos genéticos diretos razoáveis.

Palavras-chave: mestiço, leiteiro, tipo, parâmetros genéticos, Girolando

\section{Genetic Differences and Heritability Estimates for Morphological Traits in Zebus and $F_{1}$ Holstein $x$ Zebu Females}

\begin{abstract}
The objectives of this work was to evaluate possible changes in the morphological measurements among genetic groups (GG) Indubrazil, Nellore and Tabapuan and among the GG of their daughters $\mathrm{F}_{1}$ Holstein x Indubrazil (HxI), Holstein x Nellore (HxN) e Holstein $\mathrm{x}$ Tabapuan (HxT), besides the heritability estimates coefficients $\left(\mathrm{h}^{2}\right)$ for these traits. Least square method was used to verify the differences on the morphological traits among $\mathrm{GG}$ and to obtain $\mathrm{h}^{2}$ estimates from dam-daughter regression (RFM). The REML method was also used to obtain $\mathrm{h}^{2}$ estimates. Data were recorded on 183 zebu cows and 273 F1Holstein $x$ Zebu heifers. The GG of the $F_{1}$ heifers was important for its registration in the open book Girolando Association, because the GG obtained different scores for type, general appearance and dairy characteristics. For these three traits, the crossbred HxI heifers obtained higher scores than the HxN heifers; the HxT heifers obtained intermediate scores. There were no differences among the morphological traits, the width between hips and pins, the rump length, body depth and wither height and body weight for the three zebus GG and among their $\mathrm{F}_{1}$ Holstein $\mathrm{x}$ Zebu progenies. The heritability estimate changed from .09 (body depth) to .59 (ear length) by RFM and from .09 to .89 (the same traits) by REML method. The results indicated that, in a breeding system with a Holstein breed for the production of $F_{1}$ heifers to sell, the GG Indubrasil produced better characterized animals as the Girolando breed, which, possibly, will obtain higher commercial appeal than the GG Tapabuan and Nellore. Additionally, the heritability estimates indicated that the selection for morphological traits would lead to direct reasonable response.
\end{abstract}

Key Words: crossbred, dairy, type, genetic parameters, Girolando

\footnotetext{
${ }^{1}$ Parte da Dissertação apresentada à Escola de Veterinária da UFMG pelo primeiro autor, para obtenção do título de Mestre em Zootecnia. Pesquisa parcialmente financiada pelo CNPq, FAPEMIG ePRPq-UFMG.

2 Professor da Faculdade de Medicina Veterinária da Fundação de Ensino Octávio Bastos - 13870-000 - São João da Boa Vista - SP; email: mouraogb@hotmail.com

${ }^{3}$ Professor do Departamento de Zootecnia da Escola de Veterinária da UFMG, bolsista do CNPq.

${ }^{4}$ Pesquisador, Empresa de Pesquisa Agropecuária de Minas Gerais.
} 


\section{Introdução}

A produção e o comércio de fêmeas $\mathrm{F}_{1}$ Holandês $\mathrm{x}$ Zebu para produtores de leite têm sido alternativas importantes para fazendas de gado de corte. A maioria destas fêmeas mestiças são obtidas a partir de fêmeas das raças Gir e Guzerá (MADALENA, 1992). Mais recentemente, em razão da escassez numérica e dos elevados preços alcançados por estas fêmeas zebus, surgiu a alternativa de se utilizarem reprodutrizes das raças Indubrasil, Tabapuã e Nelore, além de vacas azebuadas para a produção de novilhas $\mathrm{F}_{1}$ Holandês-Zebu (MADALENA et al., 1996).

Para atender às exigências do mercado desses animais, faz-se necessária a adoção de estratégias de cruzamentos objetivando produzir fêmeas zebus que transmitam às fêmeas $\mathrm{F}_{1}$ características importantes comercialmente, como temperamento dócil, pelagem padronizada e aquelas ligadas à estrutura morfológica - úbere bem desenvolvido e orelhas maiores. Estas características são avaliadas e usadas como requisitos para classificação não apenas do controle de registro genealógico da raça Girolando, mas também dos futuros compradores.

Embora não substituam as mensurações de desempenho produtivo, as medidas lineares podem ter alguma utilidade como auxiliares no processo de escolha das fêmeas zebus que formarão a futura base genética em sistemas de cruzamento para produção de fêmeas $F_{1}$. Neste sentido, existem evidências experimentais de que algumas características morfológicas estejam genética e positivamente correlacionadas à funcionalidade, longevidade e produtividade nos rebanhos leiteiros (HONNETTE et al., 1980; BERGER et al., 1986; e CUE et al., 1990).

O objetivo deste trabalho foi averiguar possíveis variações entre algumas características morfológicas observadas em reprodutrizes de diferentes genótipos zebus e em fêmeas $\mathrm{F}_{1}$ Holandês $\mathrm{x}$ Zebu, filhas de vacas de diferentes grupos genéticos zebus, além de estimar coeficientes de herdabilidade para estas características.

\section{Material e Métodos}

Foram realizadas mensurações em 456 fêmeas, sendo 183 vacas zebus adquiridas de vários rebanhos e suas filhas, 273 novilhas $F_{1}$ Holandês x Zebu, nascidas em setembro $(n=39)$, outubro $(n=68)$, novembro $(n=38)$ e dezembro $(n=78)$ de 1993 e janeiro $(n=50)$ de 1994. As fêmeas zebus foram mensuradas em idade adulta (média de 88,3 $\pm 37,7$ meses) e classificadas, de acordo com a caracterização racial, em Indubrasil (I, n=75), Nelore (N, n=68) e Tabapuã ( $\mathrm{T}, \mathrm{n}=40$ ), denominadas neste estudo como grupos genéticos. As novilhas $\mathrm{F}_{1}$, provenientes da inseminação artificial de fêmeas zebus com sêmen de quatro touros da raça Holandesa, foram mensuradas com a idade média de 26,9 $\pm 1,4$ meses, constituindose em animais $\mathrm{F}_{1}$ Holandês $\mathrm{x}$ Indubrasil (HxI, $\mathrm{n}=110$ ), Holandês x Nelore $(\mathrm{HxN}, \mathrm{n}=106)$ e Holandês $\mathrm{x}$ Tabapuã (HxT, n=57). A aparente discrepância entre o número de vacas e novilhas foi ocasionada pelo sistema de descarte adotado na propriedade, com exclusão imediata de fêmeas vazias após a estação de acasalamento. Desta forma, considerável número de reprodutrizes, mães de novilhas $\mathrm{F}_{1}$, foi eliminado do plantel antes da realização das avaliações.

As reprodutrizes zebus não possuíam data de nascimento conhecida. Assim, as idades das mesmas foram estimadas pela cronometria dentária com precisão de 6 meses. Adicionalmente, face à grande variação de idades (de 44 a 261 meses), aliado à falta de uniformidade na distribuição das mesmas, optouse pela formação de classes de idade, da seguinte forma: reprodutrizes com idade inferior ou igual a 5,0 anos de idade como da classe $5(n=28)$; com idades de 5,5 a 6,0 anos como da classe $6(n=44)$; com idades de 6,5 a 7,0 anos como da classe $7(\mathrm{n}=58)$; com idade de 7,5 a 8,0 anos como da classe $8(n=20)$; e com idade superior a 8,0 anos como da classe $9(\mathrm{n}=33)$.

Os animais eram pertencentes à Fazenda Santa Maria, Bandeirantes Agropecuária Ltda., localizada no município de Iuiu, região semi-árida, sudoeste do Estado da Bahia. Outras informações climatológicas e a descrição sumária do manejo dos animais foram relatadas por MOURÃO (1997).

Com exceções do peso corporal e do escore de condição corporal, que foram aferidos apenas para as fêmeas $\mathrm{F}_{1}$, as seguintes mensurações morfológicas foram efetuadas em cada fêmea, incluindo as reprodutrizes zebus:

a) Altura na cernelha: distância medida entre o solo e a cernelha, estando o animal com a cabeça levantada, utilizando-se régua métrica metálica;

b) Profundidade torácica: medida realizada próxima à última vértebra torácica, com o auxílio de Bengala de Lidtyn;

c) Largura entre as tuberosidades ilíacas: distância entre as tuberosidades, medida com o auxílio de Bengala de Lidtyn;

d) Largura entre as tuberosidades isquiáticas: distância entre as tuberosidades, medida com o auxílio 
de fita métrica, com o animal contido em tronco;

e) Comprimento da garupa: distância entre a tuberosidade ilíaca e a isquiática, medida com o auxílio de fita métrica;

f) Comprimento do umbigo: distância do abdômen à extremidade da bainha do umbigo, medida com o auxílio de régua métrica metálica;

g) Comprimento da orelha: distância medida na parte dorsal entre a inserção da orelha na cabeça e a extremidade da orelha, medida com o auxílio de régua métrica metálica; e

h) Escore da condição corporal: medida subjetiva, de acordo com o sistema de avaliação da condição corporal, com escala variando de 1 (debilitado) a 9 (extremamente gordo).

As reprodutrizes zebus e as novilhas $F_{1}$ foram mensuradas nos meses de julho de 1995 e abril de 1996, respectivamente. A última ocasião coincidiu com a época de avaliação das fêmeas $\mathrm{F}_{1}$ por técnico da Associação dos Criadores da Raça Girolando (Assoleite), como exigência para expedição do registro genealógico em Livro Aberto desta raça. Estas fêmeas, submetidas ao controle de registro genealógico, foram avaliadas subjetivamente pela associação e pontuadas de acordo com aparência geral (30 pontos), caracterização leiteira (30 pontos), capacidade corporal (20 pontos) e aparelho reprodutor e aprumos (20 pontos). A totalização das pontuações atribuídas pelo técnico da Assoleite compôs nova característica, denominada classificação total para tipo (de 0 a 100 pontos).

Das 273 novilhas mestiças inicialmente avaliadas pelo técnico da Assoleite, 199 foram registradas por alcançarem padrão racial mínimo exigido para o registro genealógico em Livro Aberto da raça Girolando.

Os modelos estatísticos para ajuste e comparação entre os grupos genéticos tiveram parâmetros estimados pelo método dos quadrados mínimos. Inicialmente vários modelos foram testados para todas as características morfológicas, inclusive com a inclusão de idade do animal como variável contínua (linear e quadrática) versus variável classificatória. Da mesma forma, todas as interações simples entre os efeitos fixos foram testadas e excluídas, por não alcançarem significância $(\mathrm{P}>0,10)$. Para as características morfológicas mensuradas nas fêmeas zebus, foram considerados no modelo final os efeitos fixos de grupo genético (I, N, T) e o coeficiente de regressão linear associado à classe de idade. Para as características mensuradas nas fêmeas $\mathrm{F}_{1}$ Holandês $\mathrm{x}$ zebu, os efeitos fixos considerados no modelo final foram grupo genético (HxI, HxN e HxT), touro pai da fêmea (de um a quatro), além de idade da novilha (covariável, expressa em meses), classe de idade da reprodutriz mãe da novilha e escore da condição corporal como covariáveis.

As análises estatísticas de consistência e formação dos diferentes conjuntos de dados, estatísticas descritivas e comparações de freqüências e médias foram realizadas por intermédio do SAS (SAS, 1995). As comparações entre as médias ajustadas das características morfológicas entre os grupos zebus e, também, entre seus mestiços foram realizadas usando-se contrastes específicos. Para as comparações envolvendo informações relacionadas à freqüência de observações, o teste $\chi^{2}$ foi utilizado sobre as freqüências absolutas.

Dos quatro touros da raça Holandesa utilizados como pais das novilhas $\mathrm{F}_{1}$, três eram de origem Norte Americana, possuindo avaliações genéticas positivas para produção de leite e também para a maioria das características do perfil linear e, um de origem nacional, também com diferença esperada na progênie positiva para produção de leite (Tabela 1).

O processo de estimação dos componentes de variância por meio da regressão do valor fenotípico da filha sobre o valor fenotípico da mãe, regressão filha-mãe (RFM), considerou as mensurações realizadas nas reprodutrizes zebus, previamente ajustadas para o efeito de idade do animal, como variável independente. Além da inclusão da medida da característica na mãe, que define o coeficiente de regressão associado a $1 / 2$ da variância genética aditiva, os modelos consideraram os efeitos fixos de grupo genético ( $\mathrm{HxI}, \mathrm{HxN}$ e HxT), touro pai da fêmea $\mathrm{F}_{1}$ (de um a quatro) e idade da filha (de 24 a 29 meses). As interações simples entre os efeitos fixos, previamente testadas, foram excluídas das análises por não terem alcançaram significância $(\mathrm{P}>0,10)$. Deve-se salientar que inicialmente foram estimados quatro coeficientes de regressão pela regressão filha-mãe aninhada dentro de reprodutor (FALCONER, 1989). Como os diferentes coeficientes de regressão não diferiram entre si, optou-se pelo coeficiente único. As estimativas dos coeficientes de herdabilidade $\left(h^{2}\right)$ pelo método de regressão filha-mãe e respectivos erros-padrão das estimativas $(E P)$ foram calculados pelas expressões:

em que

$$
h^{2}=2 b
$$

$h^{2}=$ Coeficiente de herdabilidade e

$B=$ Coeficiente de regressão linear identificado no modelo de análise por $b$. 
Tabela 1 - Diferenças esperadas na progênie (DEP's) para produção de leite e características morfológicas lineares dos touros da raça Holandesa, utilizados para a produção de novilhas $F_{1}$

Table 1 - Predicted Transmiting Abilities (PTA's) for milk production and linear morphological traits of Holstein sires used for $F_{1}$ heifer production

\begin{tabular}{|c|c|c|c|c|}
\hline \multirow{2}{*}{$\begin{array}{l}\text { Característica } \\
\text { Trait } \\
\end{array}$} & \multicolumn{4}{|c|}{$\begin{array}{l}\text { DEP's dos touros }{ }^{1} \\
\text { Sire PTA's }\end{array}$} \\
\hline & 1 & 2 & 3 & 4 \\
\hline $\begin{array}{l}\text { Largura da garupa }{ }^{2} \\
\text { Hip width }\end{array}$ & $+0,7$ & $+1,6$ & $+0,6$ & - \\
\hline $\begin{array}{l}\text { Profundidade torácica }{ }^{2} \\
\text { Body depth }\end{array}$ & $+0,9$ & $+1,0$ & $+1,8$ & - \\
\hline $\begin{array}{l}\text { Altura na cernelha }{ }^{2} \\
\text { Withers height }\end{array}$ & $+0,1$ & $+1,9$ & $+1,1$ & - \\
\hline $\begin{array}{l}\text { Caracterização leiteira }{ }^{2} \\
\text { Milk type }\end{array}$ & $+0,0$ & $+1,3$ & $+2,6$ & - \\
\hline Vigor $^{2}$ & $+0,3$ & $+0,0$ & $+1,3$ & - \\
\hline $\begin{array}{l}\text { Strength } \\
\text { Tipo funcional }\end{array}$ & $+0,3$ & $+2,3$ & $+1,7$ & - \\
\hline Funcional type & & & & \\
\hline $\begin{array}{l}\text { Produção de leite, } \mathrm{kg} \\
\text { Milkproduction }\end{array}$ & +380 & +345 & +452 & +256 \\
\hline $\begin{array}{l}\text { Acurácia (\%) } \\
\text { Repeatibility }\end{array}$ & 82 & 76 & 98 & 75 \\
\hline $\begin{array}{l}{ }^{1} \text { Avaliação genética do US } \\
\text { os touros } 1,2 \text { e } 3 \text {. Avaliaçãa } \\
\text { para o touro } 4 \text {. } \\
2 \text { Classificação linear (de } \\
1 \text { Genetic evaluation from US } \\
\text { evaluation for sire } 4 \text {. }\end{array}$ & SA for & $\begin{array}{l}\text { s Uni } \\
\text { eira( }\end{array}$ & $\begin{array}{l}\text { Ia An } \\
\text { RAP }\end{array}$ & $\begin{array}{l}\text { (a para } \\
\text { NPGL) } \\
\text { genetic }\end{array}$ \\
\hline
\end{tabular}

$$
E P=2 E P_{b}
$$

em que

$E P=$ erro-padrão da herdabilidade e

$E P_{b}=$ erro-padrão do coeficiente de regressão linear.

$\mathrm{Na}$ estimação dos componentes de variância e das herdabilidades pelo método da máxima verossimilhança restrita(REML), sob modelo animal, foi utilizado o sistema MTDFREML (BOLDMAN et al., 1995). Considerou-se o seguinte modelo representado na forma matricial:

$$
\mathrm{Y}=\mathrm{X} \boldsymbol{\beta}+\mathrm{Za}+\boldsymbol{\varepsilon}
$$

em que

$\mathrm{Y}=$ vetor dos valores mensurados para a característica;

$\mathrm{X}=$ matriz de incidência dos efeitos fixos;

$\beta=$ vetor de efeitos fixos, incluindo: grupo contemporâneo, grupo genético do animal, idade;

$\mathrm{Z}$ = matriz de incidência do efeito aleatório;

$\mathrm{a}=$ vetor do efeito aleatório de animal; $\mathrm{e}$

$\varepsilon=$ vetor do efeito aleatório do erro.

A metodologia REML realiza a estimação dos componentes de variância e erros-padrão e o cálculo do coeficiente de herdabilidade segue a fórmula abaixo: em que

$$
h^{2}=\frac{\sigma_{a}^{2}}{\sigma_{a}^{2}+\sigma_{e}^{2}}
$$

$\mathrm{h}^{2}=$ coeficiente de herdabilidade;

$\sigma_{a}^{2}=$ variância genética aditiva; e

$\sigma_{e}^{2}=$ variância do erro.

Todas as análises foram realizadas utilizando-se, como valores iniciais exigidos pelo sistema, as estimativas dos componentes de variância obtidas a partir da variância fenotípica observada para a característica e da estimativa de herdabilidade obtida pelo método da regressão filha-mãe. O critério de convergência adotado considerou a iteração em que a variância dos $\mathrm{n}+1$ valores da função $2 \log \Lambda$ do Simplex fosse menor do que $10^{-9}$, sendo $n$ igual ao número de componentes de variância a serem estimados.

Faz-se importante salientar que as populações de vacas zebus e de novilhas $\mathrm{F}_{1}$ Holandês $\mathrm{x}$ Zebu utilizadas no presente estudo eram finitas e que a população de novilhas $\mathrm{F}_{1}$ não estava em equilíbrio Castle-Hardy-Weinberg.

\section{Resultados e Discussão}

\section{Estatísticas descritivas e efeito de reprodutor}

Nas Tabelas 2 e 3 estão apresentados os valores médios e desvios-padrão, mínimos e máximos, observados para as características morfológicas mensuradas, respectivamente, nas fêmeas $\mathrm{F}_{1}$ e suas mães. Como esperado, as médias observadas para comprimento da orelha e do umbigo foram maiores para vacas zebus que para suas filhas $F_{1}$ HolandêsZebu. As demais medidas morfológicas foram semelhantes para as duas categorias.

$\mathrm{Na}$ Tabela 4 são apresentadas estatísticas das avaliações feitas por técnico da Assoleite para efeito de registro genealógico das novilhas $\mathrm{F}_{1}$ como animais da raça Girolando. Observa-se que as características avaliadas pela Assoleite apresentaram variações muito pequenas (amplitudes de variação máxima de 6 pontos em escala de 30 , e de 4 pontos em escala de 20). Ainda, para as características avaliadas por técnico da Assoleite, são apresentados na Tabela 5 os coeficientes de determinação e variação dos modelos obtidos nas análises estatísticas. Todos os coeficientes de variação apresentam-se abaixo do limite inferior observado na literatura para as características avaliadas subjetivamente (escores visuais) nos animais de raças leiteiras, sendo os menores coeficientes da ordem de $10 \%$ para a característica classificação total para 
Rev. bras. zootec.

Tabela 2 - Médias observadas (Média), desvios-padrão (DP), valores mínimos (Mín) e máximos (Máx) das medidas lineares mensuradas em 273 novilhas $F_{1}$ Holandês x Zebu

Table 2 - Observed means (Mean), standard deviations (SD), minimum value (Min) and maximum values (Max) of the linear measurements made in $273 F_{1}$ Holstein $x$ Zebu heifers

\begin{tabular}{|c|c|c|c|c|}
\hline Característica & Média & DP & Min & Máx \\
\hline Trait & Mean & $S D$ & & \\
\hline $\begin{array}{l}\text { Largura entre as tuberosidades ilíacas }(\mathrm{cm}) \\
\text { Width between hips }\end{array}$ & 48,12 & 2,14 & 41,0 & 54,0 \\
\hline $\begin{array}{l}\text { Largura entre as tuberosidades isquiáticas }(\mathrm{cm}) \\
\text { Width between pins }\end{array}$ & 16,50 & 1,01 & 14,0 & 19,0 \\
\hline $\begin{array}{l}\text { Comprimento da garupa }(\mathrm{cm}) \\
\text { Rump length }\end{array}$ & 44,74 & 1,73 & 41,0 & 51,0 \\
\hline $\begin{array}{l}\text { Profundidade torácica }(\mathrm{cm}) \\
\text { Body depth }\end{array}$ & 75,64 & 4,71 & 51,0 & 88,0 \\
\hline $\begin{array}{l}\text { Altura na cernelha }(\mathrm{cm}) \\
\text { Witherheight }\end{array}$ & 132,58 & 3,45 & 125,0 & 142,0 \\
\hline $\begin{array}{l}\text { Comprimento do umbigo }(\mathrm{cm}) \\
\text { Sheath length }\end{array}$ & 3,53 & 2,02 & 0,0 & 11,0 \\
\hline $\begin{array}{l}\text { Comprimento da orelha }(\mathrm{cm}) \\
\text { Ear length }\end{array}$ & 21,95 & 2,08 & 16,0 & 27,5 \\
\hline $\begin{array}{l}\text { Escore de condição corporal (de } 1 \text { a } 9 \text { pontos) } \\
\text { Body condition score (from } 1 \text { to } 9 \text { points) }\end{array}$ & 4,98 & 0,64 & 3,0 & 6,0 \\
\hline $\begin{array}{l}\text { Peso corporal }(\mathrm{kg}) \\
\text { Body weight }\end{array}$ & 404,31 & 35,69 & 296,0 & 501,0 \\
\hline $\begin{array}{l}\text { Idade (meses) } \\
\text { Age (months) }\end{array}$ & 26,87 & 1,37 & 24,0 & 29,0 \\
\hline
\end{tabular}

Tabela 3 - Médias observadas (Média), desvios-padrão (DP), valores mínimos (Mín) e máximos (Máx) das medidas lineares mensuradas em 183 vacas Zebus

Table 3 - Observed means (Mean), standard deviations (SD), minimum values (Min) and maximum values (Max of the linear measurements made in 183 Zebu cows

\begin{tabular}{|c|c|c|c|c|}
\hline Característica & Média & $\overline{\mathrm{DP}}$ & Min & Máx \\
\hline Trait & Mean & $S D$ & & \\
\hline $\begin{array}{l}\text { Largura entre as tuberosidades ilíacas }(\mathrm{cm}) \\
\text { Width between hips }\end{array}$ & 50,18 & 2,77 & 41,0 & 59,0 \\
\hline $\begin{array}{l}\text { Largura entre as tuberosidades isquiáticas }(\mathrm{cm}) \\
\text { Width between pins }\end{array}$ & 15,86 & 1,70 & 12,0 & 22,0 \\
\hline $\begin{array}{l}\text { Comprimento da garupa }(\mathrm{cm}) \\
\text { Rump length }\end{array}$ & 45,47 & 2,51 & 39,0 & 51,0 \\
\hline $\begin{array}{l}\text { Profundidade torácica }(\mathrm{cm}) \\
\text { Body depth }\end{array}$ & 76,11 & 4,15 & 56,0 & 86,0 \\
\hline $\begin{array}{l}\text { Altura na cernelha }(\mathrm{cm}) \\
\text { Wither height }\end{array}$ & 132,66 & 4,98 & 121,0 & 152,0 \\
\hline $\begin{array}{l}\text { Comprimento do umbigo }(\mathrm{cm}) \\
\text { Sheath length }\end{array}$ & 7,39 & 3,16 & 0,5 & 17,0 \\
\hline $\begin{array}{l}\text { Comprimento da orelha }(\mathrm{cm}) \\
\text { Ear length }\end{array}$ & 28,83 & 3,93 & 21,0 & 40,5 \\
\hline $\begin{array}{l}\text { Idade (meses) } \\
\text { Age (months) }\end{array}$ & 88,32 & 37,67 & 44,0 & 261,0 \\
\hline
\end{tabular}

tipo. Classificações para todas as outras características do perfil linear apresentam na literatura coeficientes de variação acima de 20\% (MEYER et al., 1987; BROTHERSTONE e HILL, 1991). Entretanto, VIJ et al. (1990), em estudo com características do perfil linear em animais da raça Tharparkar, relataram coeficientes abaixo de $10 \%$ para a maioria das medidas avaliadas. A pequena variação em torno da média observada no presente estudo ocorreu, provavelmente, em razão de as avaliações terem sido feitas por apenas um técnico credenciado, além da falta de utilização dos extremos da escala adotada e da tendência de se atribuirem notas dentro de pequena faixa de valores.

Os coeficientes de determinação encontrados no presente estudo para características do perfil linear 
MOURÃO et al.

Tabela 4 - Médias observadas (Média), desvios-padrão (DP), valores mínimos (Mín.) e máximos (Máx.) das características avaliadas subjetivamente, presentes no registro genealógico de 191 novilhas $F_{1}$ Holandês $x$ Zebu, realizada pela Assoleite, por ocasião da expedição do registro em livro aberto

Table 4 - Observed means (Mean), standard deviations (SD), minimum values (Min) and maximum values (Max) of the traits evaluated subjectively, presented in the genealogical register of $191 F_{1}$ Holstein $x$ Zebu heifers, realized by Assoleite, by the time of the expedition of the open book registration

\begin{tabular}{|c|c|c|c|c|}
\hline Característica & Média & $\mathrm{DP}$ & Min & Máx \\
\hline Trait & Mean & $S D$ & & \\
\hline Aparência geral (de 0 a 30 pontos) & 26,61 & 1,03 & 23,0 & 29,0 \\
\hline General appearance (from 0 to 30 points) & & & & \\
\hline $\begin{array}{l}\text { Caracterização leiteira (de } 0 \text { a } 30 \text { pontos) } \\
\text { Dairy characterization (from } 0 \text { to } 30 \text { points) }\end{array}$ & 26,73 & 0,97 & 23,0 & 29,0 \\
\hline $\begin{array}{l}\text { Capacidade corporal (de } 0 \text { a } 20 \text { pontos) } \\
\text { Body capacity (from } 0 \text { to } 20 \text { points) }\end{array}$ & 14,88 & 0,32 & 14,0 & 15,0 \\
\hline $\begin{array}{l}\text { Aparelho reprodutor e aprumos (de } 0 \text { a } 20 \text { pontos) } \\
\text { Reproduction system, feet and legs (from } 0 \text { to } 20 \text { points) }\end{array}$ & 12,03 & 0,32 & 11,0 & 15,0 \\
\hline $\begin{array}{l}\text { Classificação total para tipo (de } 0 \text { a } 100) \\
\text { Total type score (from } 0 \text { to } 100 \text { points) }\end{array}$ & 80,25 & 2,17 & 72,0 & 85,0 \\
\hline
\end{tabular}

Tabela 5 - Médias de mínimos quadrados e erros-padrão (MQM $\pm E P)$, coeficientes de determinação $\left(R^{2}\right.$, \%) e coeficientes de variação (CV, \%) para as características de tipo avaliadas em novilhas $\mathrm{F}_{1}$ Holandês $\mathrm{x}$ Zebu

Table 5 - Least square means and standard errors ( $L S M \pm S E)$, coefficients of determination $\left(R^{2}\right)$ and coefficients of variation (CV) for type traits evaluated in $F_{1}$ Holstein $x$ Zebu heifers

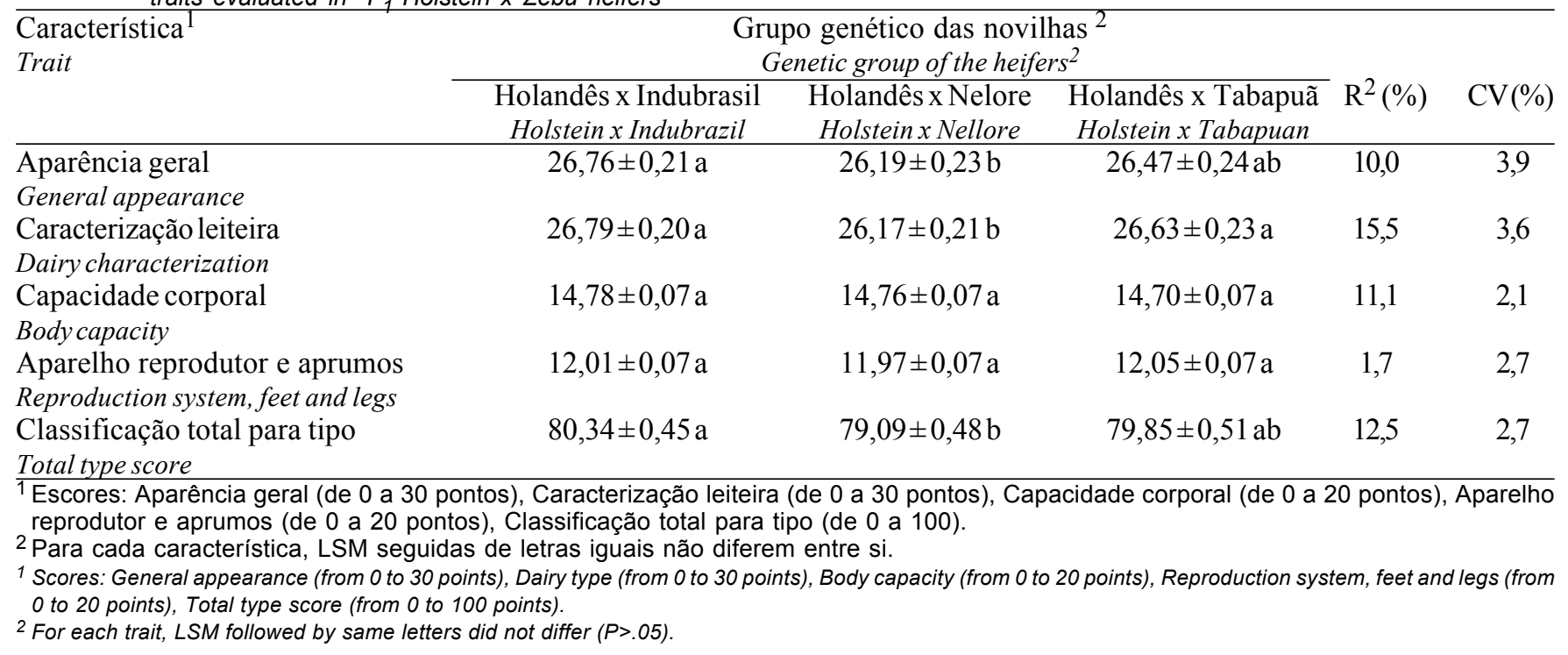

podem ser considerados dentro dos limites encontrados na literatura, à exceção do obtido para aparelho reprodutor e aprumos, que se apresentou muito baixo (1,7\%, Tabela 5). Segundo VIJ et al. (1990), os efeitos do estádio fisiológico não considerados no presente estudo podem ser fontes de variação importantes.

$\mathrm{Na}$ Tabela 8 são listados os coeficientes de variação e determinação dos modelos envolvendo mensurações morfológicas de fêmeas $\mathrm{F}_{1}$. Todos os coeficientes de variação foram abaixo de $10 \%$. De acordo com a literatura, todas as características mensuradas com procedimentos objetivos apresentam coeficientes de variação próximos de $10 \%$
(GILBERT et al., 1993 a,b; MAGNABOSCO et al., 1996). No presente estudo, o maior coeficiente de variação foi observado para comprimento do umbigo $(54,9 \%)$, possivelmente em razão da dificuldade de se fazer esta mensuração. Alternativa viável para a mensuração desta característica pode ter sido a utilização de avaliação subjetiva com escores visuais, apesar de apresentar coeficientes de variação da ordem de 20 a $40 \%$ (KLEI et al., 1988; BROTHERSTONE e HILL, 1991; e SHORT e LAWLOR, 1992).

Nota-se que, apesar das diferenças entre DEP para características do perfil linear dos três touros 
Holandeses importados utilizados neste estudo (Tabela 1), não foram observados efeitos $(\mathrm{P}>0,10)$ do reprodutor pai da novilhas $\mathrm{F}_{1}$ sobre as características aparência geral, caracterização leiteira, aparelho reprodutor e aprumos e classificação total para tipo.

O efeito fixo da variável reprodutor foi importante $(\mathrm{P}<0,05)$ para as características largura entre tuberosidades ilíacas, comprimentos do umbigo e da orelha, peso corporal e capacidade corporal (avaliação subjetiva por técnico da Assoleite). Entre essas, as variações entre filhas dos diferentes reprodutores para as características comprimento do umbigo e da orelha foram inesperadas, pois não se conhece nenhum trabalho sobre controle e seleção destas características em rebanhos da raça Holandesa. Resultado semelhante foi observado por ALENCAR et al. (1994), em relato sobre estimativas do coeficiente de herdabilidade para comprimento de umbigo em fêmeas da raça Canchim, sendo o efeito de reprodutor altamente significativo.

\section{Diferenças entre os grupos genéticos}

Como mencionado anteriormente, do ponto de vista comercial, as fêmeas mestiças registradas como Girolando, mesmo que em livro aberto, são mais valorizadas no mercado que fêmeas não-registradas. Faz-se, então, necessário o enquadramento das características morfológicas ao padrão racial da raça Girolando para que se possa elevar a lucratividade dos sistemas de produção de fêmeas $\mathrm{F}_{1}$. Para o grupo em estudo, $72,9 \%$ das fêmeas obtiveram registro. $\mathrm{Na}$ Tabela 6 são apresentadas as porcentagens de novilhas que alcançaram o padrão fenotípico exigido para o registro em livro aberto pela Assoleite, de acordo com o grupo genético. Nota-se que as novilhas $\mathrm{F}_{1}$ $\mathrm{HxN}$ apresentaram porcentagem de registros muito abaixo (55,7\%; $\mathrm{P}<0,001)$ que novilhas filhas de vacas Indubrasil $(84,5 \%)$ e Tabapuã $(82,5 \%)$. Entre esses dois últimos grupos genéticos não houve diferença na percentagem de fêmeas registradas.

Entre as características avaliadas pela Assoleite, fêmeas mestiças $\mathrm{F}_{1} \mathrm{HxI}$ alcançaram pontuações superiores às $\mathrm{HxN}$ na aparência geral, caracterização leiteira e classificação total para tipo. As fêmeas $\mathrm{F}_{1} \mathrm{HxT}$ também foram superiores às $\mathrm{HxN}$ para a caracterização leiteira, e não diferiram das HxI e das $\mathrm{HxN}$ para as características aparência geral e classificação total para tipo. Ainda, na avaliação da caracterização leiteira, a semelhança entre fêmeas mestiças $F_{1}$ filhas de vacas zebus dos grupos Indubrasil e Tabapuã foi, de certa forma, inesperada. Esperavam-se pontuações maiores para as mestiças de HxI em relação às HxT, pois no desenvolvimento da raça Indubrasil houve maior contribuição da raça Gir. Assim, animais da raça Indubrasil, e não da Tabapuã, têm maior semelhança fenotípica com animais Gir. Além disto, sempre houve maior ênfase no uso de fêmeas Indubrasil para a produção leiteira na região de origem das mesmas. Para as características capacidade corporal e aparelho reprodutor e aprumos não foram observadas diferenças entre os três grupos genéticos (Tabela 5).

O rebanho base do experimento era constituído por fêmeas zebus provenientes de outros criatórios. Entretanto, no momento da escolha e compra desses animais foram considerados aspectos fenotípicos empíricos, buscando e, aparentemente, conseguindo, certa uniformidade para as características estudadas (Tabela 7). Dessa forma, as análises estatísticas das diversas características morfológicas revelaram que as reprodutrizes Indubrasil, Nelore e Tabapuã foram

Tabela 6 - Freqüências (porcentagens) de novilhas $F_{1}$ registradas e não registradas em livro aberto no livro de registro genealógico da raça Girolando, de acordo com seu grupo genético

Table 6 - Frequency ( percents) of $F_{1}$ Holstein $x$ Zebu heifers registered and not registered in open book in the genealogical register book of the Girolando breed, in accordance of genetic group

\begin{tabular}{lccc}
\hline $\begin{array}{l}\text { Novilhas } \\
\text { Heifers }\end{array}$ & \multicolumn{3}{c}{$\begin{array}{c}\text { Grupo genético das novilhas } \\
\text { Genetic group of the heifers }\end{array}$} \\
\cline { 2 - 4 } & $\begin{array}{c}\text { Holandês x Indubrasil } \\
\text { Holstein } x \text { Indubrazil }\end{array}$ & $\begin{array}{c}\text { Holandês x Nelore } \\
\text { Holstein } x \text { Nellore }\end{array}$ & $\begin{array}{c}\text { Holandês x Tabapuã } \\
\text { Holstein } x \text { Tabapuan }\end{array}$ \\
\hline $\begin{array}{l}\text { Registradas } \\
\text { Registered }\end{array}$ & $93(84,5) \mathrm{a}$ & $59(55,7) \mathrm{b}$ & $47(82,5) \mathrm{a}$ \\
$\begin{array}{l}\text { Não registradas } \\
\text { Notregistered } \\
\text { Total }\end{array}$ & $17(15,5) \mathrm{a}$ & $47(44,3) \mathrm{b}$ & $10(17,5) \mathrm{a}$ \\
Total $(\%)$
\end{tabular}

${ }^{1}$ Freqüências, na linha, seguidas de letras diferentes diferem entre si $\left(\chi^{2}=25,62 ; P<0,001\right)$.

${ }^{1}$ Frequencies, in a row, followed by same letters did not differ $\left(\chi^{2}=25.62 ; P<.001\right)$. 
MOURÃO et al.

Tabela 7 - Médias de mínimos quadrados e erros padrão (MQM $\pm E P)$, coeficientes de determinação $\left(R^{2}\right)$ e coeficientes de variação $(\mathrm{CV})$ para as características morfológicas mensuradas em vacas Zebus

Table 7 - Least square means and standard errors (LSM $\pm S E)$, coefficients of determination $\left(R^{2}\right)$ and coefficients of variation (CV) for the morphological traits made in Zebu cows

\begin{tabular}{|c|c|c|c|c|c|}
\hline \multirow{3}{*}{$\begin{array}{l}\text { Característica }{ }^{1} \\
\text { Trait }\end{array}$} & \multirow{2}{*}{\multicolumn{3}{|c|}{$\begin{array}{l}\text { Grupo genético das novilhas } \\
\text { Genetic group of the heifers }\end{array}$}} & \multirow[b]{3}{*}{$\begin{array}{r}\mathrm{R}^{2} \\
(\%)\end{array}$} & \multirow[b]{3}{*}{$\begin{array}{l}\mathrm{CV} \\
(\%)\end{array}$} \\
\hline & & & & & \\
\hline & $\begin{array}{l}\text { Indubrasil } \\
\text { Indubrazil }\end{array}$ & $\begin{array}{l}\text { Nelore } \\
\text { Nellore }\end{array}$ & $\begin{array}{c}\text { Tabapuã } \\
\text { Tabapuan }\end{array}$ & & \\
\hline Largura entre as tuberosidades ilíacas $(\mathrm{cm})$ & $50,66 \pm 0,31 \mathrm{a}$ & $49,66 \pm 0,33 a$ & $50,16 \pm 0,43 a$ & 5,8 & 5,4 \\
\hline $\begin{array}{l}\text { Width between hips } \\
\text { Largura entre as tuberosidades isquiáticas }(\mathrm{cm})\end{array}$ & $16,09 \pm 0,20 \mathrm{a}$ & $15,68 \pm 0,21 \mathrm{a}$ & $15,74 \pm 0,27 \mathrm{a}$ & 1,1 & 10,7 \\
\hline Width between pins & & & & & \\
\hline $\begin{array}{l}\text { Comprimento da garupa }(\mathrm{cm}) \\
\text { Rump length }\end{array}$ & $45,46 \pm 0,29 a$ & $45,51 \pm 0,30 \mathrm{a}$ & $45,36 \pm 0,40 \mathrm{a}$ & 2,8 & 5,5 \\
\hline $\begin{array}{l}\text { Profundidade torácica }(\mathrm{cm}) \\
\text { Body depth }\end{array}$ & $76,63 \pm 0,48 a$ & $75,60 \pm 0,50 \mathrm{a}$ & $75,94 \pm 0,66 a$ & 1,2 & 5,5 \\
\hline $\begin{array}{l}\text { Altura na cernelha }(\mathrm{cm}) \\
\text { Wither height }\end{array}$ & $132,79 \pm 0,58 \mathrm{a}$ & $132,39 \pm 0,61 \mathrm{a}$ & $132,79 \pm 0,79 a$ & 0,9 & 3,8 \\
\hline $\begin{array}{l}\text { Comprimento do ubigo }(\mathrm{cm}) \\
\text { Sheath length }\end{array}$ & $7,84 \pm 0,36 \mathrm{a}$ & $6,68 \pm 0,38 \mathrm{a}$ & $7,77 \pm 0,49 \mathrm{a}$ & 3,1 & 42,4 \\
\hline $\begin{array}{l}\text { Comprimento da orelha }(\mathrm{cm}) \\
\text { Ear length }\end{array}$ & $31,59 \pm 0,36 \mathrm{a}$ & $26,38 \pm 0,38 \mathrm{c}$ & $27,80 \pm 0,50 \mathrm{~b}$ & 37,5 & 10,9 \\
\hline
\end{tabular}

semelhantes fenotipicamente, com exceção da característica comprimento da orelha, para a qual fêmeas do grupo genético Indubrasil tiveram, em média, 5,21 e 3,79 $\mathrm{cm}$ mais que fêmeas Nelore e Tabapuã, respectivamente $(\mathrm{P}<0,05)$. Vacas Tabapuã, por sua vez, tiveram orelhas $1,42 \mathrm{~cm}$ maiores que Nelores $(\mathrm{P}<0,05)$. Estas diferenças estão de acordo com os padrões fenotípicos das três raças.

A Tabela 8 inclui as médias ajustadas, os errospadrão e o resultado das comparações de médias para as características morfológicas em fêmeas $F_{1}$ Holandês x Zebu. Como era esperado, filhas de reprodutrizes do grupo Indubrasil, HxI apresentaram comprimento de orelha $(22,38 \mathrm{~cm})$ maiores que as do grupo $\mathrm{HxT}(21,34 \mathrm{~cm})$, e essas maiores que as do grupo $\mathrm{HxN}(20,59 \mathrm{~cm} ; \mathrm{P}<0,05)$. Estas diferenças certamente estão ligadas às diferenças observadas nas progenitoras. Observou-se também diferença $(\mathrm{P}<0,05)$ entre o comprimento de umbigo das novilhas $\mathrm{HxT}(4,02 \mathrm{~cm})$ e os das novilhas $\mathrm{HxI}(3,10 \mathrm{~cm})$ e $\mathrm{HxN}(3,08 \mathrm{~cm})$. Para as demais características não foram observadas diferenças entre os grupos genéticos estudados.

\section{Coeficientes de herdabilidade}

As estimativas dos coeficientes herdabilidade pelos métodos RFM e REML e os coeficientes de determinação e variação são apresentados na Tabela 9.

A presença de coeficientes de variação relativamente baixos para a maioria das características contrasta com as informações da literatura, em que os mesmos se situam acima de 7\% (GILBERT et al.,
1993 a,b; MAGNABOSCO et al., 1996). Possível justificativa seria a maneira como as mensurações foram realizadas nos diferentes estudos. No presente trabalho, diferente de outros, apenas um técnico fez todas as mensurações, que foram realizadas com os animais contidos no tronco. Novamente, o elevado coeficiente de variação observado para a característica comprimento de umbigo $(47,39 \%)$ refletiu a maior dificuldade associada à tomada dessa medida.

Todos os coeficientes de determinação foram muito baixos, evidenciando que o modelo explicou pequena fração da variação total, ou seja, existiam outros fatores atuando sobre as variáveis. O maior coeficiente de determinação foi observado para comprimento da orelha, o qual pode ser atribuído, em parte, à pequena influência do meio ambiente sobre esta característica.

Os coeficientes de herdabilidade estimados por meio dos métodos RFM e REML para as características largura entre as tuberosidades ilíacas $(0,36 \mathrm{e}$ 0,51 , respectivamente), largura entre as tuberosidades ísquiáticas $(0,41$ e 0,54 , respectivamente), comprimento de garupa $(0,26$ e 0,54 , respectivamente) e altura na cernelha $(0,28$ e 0,33 , respectivamente) foram de moderada a alta magnitude, estando dentro da variação encontrada na literatura (CUE et al., 1990; SHORT e LAWLOR, 1992; WEIGEL et al., 1995; e FERNANDES et al., 1996). Entretanto, as estimativas obtidas em raças puras não são necessariamente comparáveis às obtidas em resultados de 
52 Rev. bras. zootec.

Tabela 8 -Médias de mínimos quadrados e erros padrão (MQM $\pm E P)$, coeficientes de determinação $\left(R^{2}\right)$ e coeficientes de variação $(\mathrm{CV})$ para as características morfológicas mensuradas em novilhas $\mathrm{F}_{1}$ Holandês $\mathrm{x}$ Zebu

Table 8 - Least square means and standard errors (LSM $\pm S E$ ), coefficients of determination ( $R^{2}$ ) and coefficients of variation (CV) to morphological traits made in $F_{1}$ Holstein $x$ Zebu heifers

\begin{tabular}{|c|c|c|c|c|c|}
\hline \multirow{3}{*}{$\begin{array}{l}\text { Característica }{ }^{1} \\
\text { Trait }\end{array}$} & \multirow{2}{*}{\multicolumn{3}{|c|}{$\begin{array}{c}\text { Grupo genético das novilhas } \\
\text { Genetic group of the heifers }\end{array}$}} & \multirow[b]{3}{*}{$\mathrm{R}^{2}(\%)$} & \multirow[b]{3}{*}{$\mathrm{CV}(\%)$} \\
\hline & & & & & \\
\hline & $\begin{array}{l}\text { Holandês x Indubrasil } \\
\text { Holstein x Indubrazil }\end{array}$ & $\begin{array}{l}\text { Holandês x Nelore } \\
\text { Holstein } x \text { Nellore }\end{array}$ & $\begin{array}{l}\text { Holandês x Tabapuã } \\
\text { Holstein x Tabapuan }\end{array}$ & & \\
\hline $\begin{array}{l}\text { Largura entre as tuberosidades ilíacas }(\mathrm{cm}) \\
\text { Width between hips }\end{array}$ & $48,06 \pm 0,26^{\mathrm{a}}$ & $48,04 \pm 0,24 \mathrm{a}$ & $48,19 \pm 0,33 \mathrm{a}$ & 19,4 & 4,1 \\
\hline $\begin{array}{l}\text { Largura entre as tuberosidades isquiáticas }(\mathrm{cm}) \\
\text { Width between pins }\end{array}$ & $16,34 \pm 0,13^{\mathrm{a}}$ & $16,46 \pm 0,12 \mathrm{a}$ & $16,53 \pm 0,17 \mathrm{a}$ & 3,0 & 6,1 \\
\hline $\begin{array}{l}\text { Comprimento da garupa }(\mathrm{cm}) \\
\text { Rump length }\end{array}$ & $44,60 \pm 0,22^{\mathrm{a}}$ & $44,98 \pm 0,21 \mathrm{a}$ & $45,23 \pm 0,28 \mathrm{a}$ & 7,7 & 3,8 \\
\hline $\begin{array}{l}\text { Profundidade torácica }(\mathrm{cm}) \\
\text { Body depth }\end{array}$ & $75,64 \pm 0,57 \mathrm{a}$ & $75,05 \pm 0,54 a$ & $76,08 \pm 0,73 a$ & 15,0 & 5,8 \\
\hline $\begin{array}{l}\text { Altura na cernelha }(\mathrm{cm}) \\
\text { Wither height }\end{array}$ & $132,33 \pm 0,45 \mathrm{a}$ & $132,20 \pm 0,43 a$ & $132,89 \pm 0,57 \mathrm{a}$ & 3,2 & 2,6 \\
\hline $\begin{array}{l}\text { Comprimento do umbigo }(\mathrm{cm}) \\
\text { Sheath length }\end{array}$ & $3,10 \pm 0,25 \mathrm{a}$ & $3,08 \pm 0,24 \mathrm{a}$ & $4,02 \pm 0,32 \mathrm{a}$ & 9,8 & 54,9 \\
\hline $\begin{array}{l}\text { Comprimento da orelha }(\mathrm{cm}) \\
\text { Ear length }\end{array}$ & $22,38 \pm 0,24 \mathrm{a}$ & $20,59 \pm 0,23 \mathrm{c}$ & $21,34 \pm 0,30 \mathrm{~b}$ & 25,8 & 8,3 \\
\hline $\begin{array}{l}\text { Peso corporal }(\mathrm{kg}) \\
\text { Bodyweight }\end{array}$ & $403,50 \pm 3,89 a$ & $398,80 \pm 3,71 \mathrm{a}$ & $407,69 \pm 4,91 \mathrm{a}$ & 34.6 & 7,3 \\
\hline
\end{tabular}

${ }^{1}$ Para cada característica, LSM seguidas de letras iguais não diferiram.

${ }^{1}$ For each trait, LSM followed by the same letters did not differ.

Tabela 9 - Coeficientes de herdabilidade $\left(\mathrm{h}^{2}\right)$ e erros padrão (EP) de características morfológicas estimados por dois métodos (regressão filha-mãe e REML), coeficientes de determinação $\left(R^{2}\right)$ e coeficientes de variação (CV) observados nas análises de regressão filha-mãe

Table 9 - Coefficients of heritability $\left(h^{2}\right)$ and standard errors (SE) of morphological traits estimated by two methods (dam- daughter regression and REML), coefficients of determination $\left(R^{2}\right)$ and coefficients of variation (CV) observed in the analysis of damdaughter regression

\begin{tabular}{|c|c|c|c|c|}
\hline \multirow[b]{2}{*}{$\begin{array}{l}\text { Característica } \\
\text { Trait }\end{array}$} & \multicolumn{3}{|c|}{$\begin{array}{l}\text { Método e estimativas (\%) } \\
\text { Method and estimates } \\
\text { Regressão filha-mãe } \\
\text { Dam-daughter regression }\end{array}$} & \multirow{2}{*}{$\begin{array}{c}\text { REML }^{1} \\
\mathrm{~h}^{2}\end{array}$} \\
\hline & $\begin{array}{c}\mathrm{h}^{2} \pm \mathrm{EP} \\
h^{2} \pm S E\end{array}$ & $\mathrm{R}^{2}(\%)$ & $\mathrm{CV}(\%)$ & \\
\hline $\begin{array}{l}\text { Largura entre as tuberosidades ilíacas (cm) } \\
\text { Width between hips }\end{array}$ & $0,36 \pm 0,12$ & 20,63 & 4,22 & 0,51 \\
\hline $\begin{array}{l}\text { Largura entre as tuberosidades isquiáticas }(\mathrm{cm}) \\
\text { Width between pins }\end{array}$ & $0,41 \pm 0,09$ & 14,42 & 5,95 & 0,54 \\
\hline $\begin{array}{l}\text { Comprimento da garupa }(\mathrm{cm}) \\
\text { Rump length }\end{array}$ & $0,26 \pm 0,10$ & 16,25 & 3,68 & 0,54 \\
\hline $\begin{array}{l}\text { Profundidade torácica }(\mathrm{cm}) \\
\text { Bodydepth }\end{array}$ & $0,09 \pm 0,17$ & 8,60 & 5,97 & 0,09 \\
\hline $\begin{array}{l}\text { Altura na cernelha }(\mathrm{cm}) \\
\text { Wither height }\end{array}$ & $0,28 \pm 0,11$ & 10,08 & 2,55 & 0,33 \\
\hline $\begin{array}{l}\text { Comprimento do umbigo }(\mathrm{cm}) \\
\text { Sheath length }\end{array}$ & $0,48 \pm 0,09$ & 26,10 & 47,39 & 0,70 \\
\hline $\begin{array}{l}\text { Comprimento da orelha }(\mathrm{cm}) \\
\text { Ear length }\end{array}$ & $0,59 \pm 0,08$ & 51,41 & 6,76 & 0,89 \\
\hline
\end{tabular}

cruzamentos, como os do presente trabalho.

Profundidade torácica apresentou coeficientes de herdabilidade muito baixos $(0,09$ pelos dois métodos), sendo esse valor menor que os relatados por
MUKAI et al. (1993) para fêmeas da raça Japanese Black, de 0,19 (profundidade torácica) e 0,14 (circunferência torácica) estimadas pelo método REML, e menores ainda que 0,42 (profundidade corporal em fême- 
as da raça Holandesa) e 0,49 (perímetro torácico em animais da raça Brahman) citados por MEYER et al. (1987) e FERNANDES et al. (1996), respectivamente.

As herdabilidades estimadas para comprimento do umbigo foram altas, 0,48 (RFM) e 0,70 (REML), estando dentro da magnitude encontrada por ALENCAR et al. (1994) para fêmeas da raça Canchim, 0,75 $\pm 0,25$, utilizando estrutura familiar de meio irmãs paternas. Para comprimento da orelha as estimativas também foram altas 0,59 (RFM) e 0,89 (REML). Não foram encontradas citações sobre estimativas de herdabilidade para esta característica.

Possível explicação para as diferenças entre os valores das estimativas de herdabilidade a partir dos dois métodos seria a utilização da matriz de parentesco no método REML (VAN VLECK e HUDSON (1982); JOHNSON et al.,1992). Neste trabalho, as estimativas obtidas pelo método REML foram, em geral, maiores, quando todas as informações conhecidas de parentesco são consideradas, encontrando, portanto, suporte na literatura (VAN VLECK e HUDSON, 1982; JOHNSON et al.,1992).

\section{Conclusões}

O grupo genético das fêmeas $F_{1}$ foi importante para o sucesso na avaliação fenotípica e no registro genealógico em Livro Aberto da raça Girolando. Nessas avaliações oficiais, os diferentes grupos genéticos tiveram pontuações diferentes para as características classificação total para tipo, aparência geral e caracterização leiteira, nas quais as mestiças Holandês x Indubrasil foram consideradas melhor caracterizadas que as Holandês x Nelore. As características morfológicas ligadas à estrutura corporal, entre elas as larguras entre as tuberosidades ilíacas e isquiáticas, o comprimento da garupa, a profundidade torácica, a altura na cernelha e o peso corporal apresentaram-se muito homogêneos entre os três grupos raciais zebus e entre suas progênies $F_{1}$ Holandês $x$ Zebu.

$\mathrm{O}$ efeito significativo do reprodutor da raça Holandesa sobre as características comprimento de umbigo e de orelha foi inesperado, assim, ensaios que envolvam amostras com maiores números de touros necessitam ser feitos para inferências mais seguras.

\section{Agradecimentos}

À Bandeirantes Agropecuária Ltda., em nome do Zootecnista Lúcio H. Machado, pela cessão da estrutura física da Fazenda Santa Maria e colaboração durante a coleta das informações.

À Pró-Reitoria de Pesquisa da Universidade Federal de Minas Gerais (PRPq-UFMG), à Fundação de Amparo à Pesquisa do Estado de Minas Gerais (FAPEMIG) e ao Conselho Nacional de Desenvolvimento Científico e Tecnológico ( $\mathrm{CNPq}$ ), por viabilizarem este estudo.

\section{Referências Bibliográficas}

ALENCAR, M.M., CORREA, L.A., TULliO, R.R. Herdabilidade do tamanho de umbigo em fêmeas da raça Canchim. In: REUNIÃO ANUAL DA SOCIEDADE BRASILEIRA DE ZOOTECNIA, 31, 1994, Maringá, PR, SBZ, Anais... Maringá: SBZ, p. 159, 1994.

BERGER, E.B., DISHMAN, W.A., FREEMAN, A.E. 1986. Evaluation of merit of a corrective mating program for Holstein cattle. J. Dairy Sci., 69(5):1403-1410.

BOLDMAN, K.G., KRIESE, L.A., Van VLECK, L.D. et al. 1995. A manual for Use of MTDFREML. A set of programs to obtain estimates of variances and covariances (DRAFT). USDA, Agricultural Research Service. 115 p.

BROTHERSTONE, S. e HILL, W.G. 1991. Dairy herd life in relation to linear type traits and production. 1.Phenotypic and genetic analyses in pedigree type classified herds. Anim. Prod., 53(3):279-287.

CUE, R.I., MONARDES, G.H., HAYES, J.F. 1990. Relationships of calving ease with type traits. J. Dairy Sci., 73(12):35863590 .

FALCONER, D. S. 1989. Introdution to Quantitative Genetics. 3.ed. New York: Longman Group. 437p.

FERNANDES, A., MAGNABOSCO, C.U., OJALA, M. et al. Estimativas de parâmetros genéticos e ambientais de medidas corporais e peso me bovinos da raça Brahman nos trópicos. In: REUNIÃO ANUAL DA SOCIEDADE BRASILEIRA DE ZOOTECNIA, 33, 1996, Fortaleza, CE. Anais... Fortaleza: SBZ, p. 65-67, 1996.

GILBERT, R.P., BAILEY, D.R.C., SHANNON, N.H. 1993 a. Body dimensions and carcass measurements of cattle selected for postweaning gain fed two different diets. J. Anim. Sci., 71:1688-1698.

GILBERT, R.P., BAILEY, D.R.C., SHANNON, N.H. 1993b. Linear body measurements of cattle before and after 20 years of selection for postweaning gain when fed two different diets. J. Anim. Sci., 71:1712-1720.

HONNETTE, J.E., VINSON, W.E., WHITE, J.M. et al. 1980. Contributions of descriptively coded type traits to longevity of Holstein cows. J. Dairy Sci., 63(5):807-815.

JOHNSON, Z.B., WRIGHT, D.W., BROWN, C.J. 1992. et al. Effect of including relationships in the estimation of genetic parameters of beef calves. J. Anim. Sci., 70(1):78-88.

KLEI, L., POLLAK, E.J., QUAAS, R.L. 1988. Genetic and environmental parameters associated with linearized type appraisal scores. J. Dairy Sci., 71(10):2744-2752.

MADALENA, F.E. 1992. Reposição com novilhas $\mathrm{F}_{1}$ : Um esquema simples de cruzamento. Inf. Agropec., 16(177):23-25.

MADALENA, F.E., ABREU, C.P, SAMPAIO, I.B.M. et al. 1996. Cruzamentos entre raça leiteiras: O que faz o produtor? Cad. Tec. Esc. Vet. UFMG, (18):7-9.

MAGNABOSCO, C.U., OJALA, M., FERNANDES, A. et al. Efeitos de fatores ambientais sobre medidas corporais e peso em bovinos da raça Brahman no Mexico. In: REUNIÃO 
54 Rev. bras. zootec.

ANUAL DA SOCIEDADE BRASILEIRA DE ZOOTECNIA, 33, 1996, Fortaleza, CE. Anais.. Fortaleza: SBZ, p. 1-3, 1996.

MEYER, K., BROTHERSTONE, S., HILL, W.G. et al. 1987. Inheritance of linear type traits in dairy cattle and correlations with milk production. Anim. Prod., 44(1):1-10.

MOURÃO, G.B. Temperamento e características morfológicas em fêmeas Zebus e $F_{1}$ Holandês $x$ Zebu. Escola de Veterinária: UFMG, 113 p., 1997. Dissertação (Mestrado em Zootecnia) Universidade Federal de Minas Gerais, 1997.

MUKAI, F., OKANISHI, T., YOSHIMURA, T. 1993. Genetic relationships between body measurements of heifers and carcass traits of fattening cattle in Japanese Black. Anim. Sci. Technol. (Jpn.), 64(9):865-872.

SAS - Statistical Analyses Systems User's Guide for Windows: Statistics., Cary., NC., SAS Institute Inc., 1995 (Versão 6.09).

SHORT, T.H., LAWLOR, T.J. 1992. Genetic parameters of conformation traits, milk yield, and herd life in Holsteins. J. Dairy Sci., 75(7):1987-1998.
VAN VLECK, L.D., HUDSON, G.F.S. 1982. Relationships among sires in estimating genetic variance. J. Dairy Sci., 65(8):1663-1665.

VIJ, P.K., BALAIN, D.S., GEORGE, M. et al. 1990. Linear type traits and their influence on milk production in Tharparkar cattle. Ind. J. Anim. Sci., 60(7):845-852.

WEIGEL, D.J., CASSELL, B.G., HOESCHELE, I. et al. 1995. Multiple trait prediction of transmitting abilities for herd life and estimation of economic weights using relative net income adjusted for opportunity cost. J. Dairy Sci., 78(3):639-647.

Recebido em: 08/05/98

Aceito em: 17/08/98 